\title{
Metal water bottle causing tongue entrapment in a child
}

\author{
Vinay T. Fernandes MD, Elaine Ng MD, Paolo Campisi MD
}
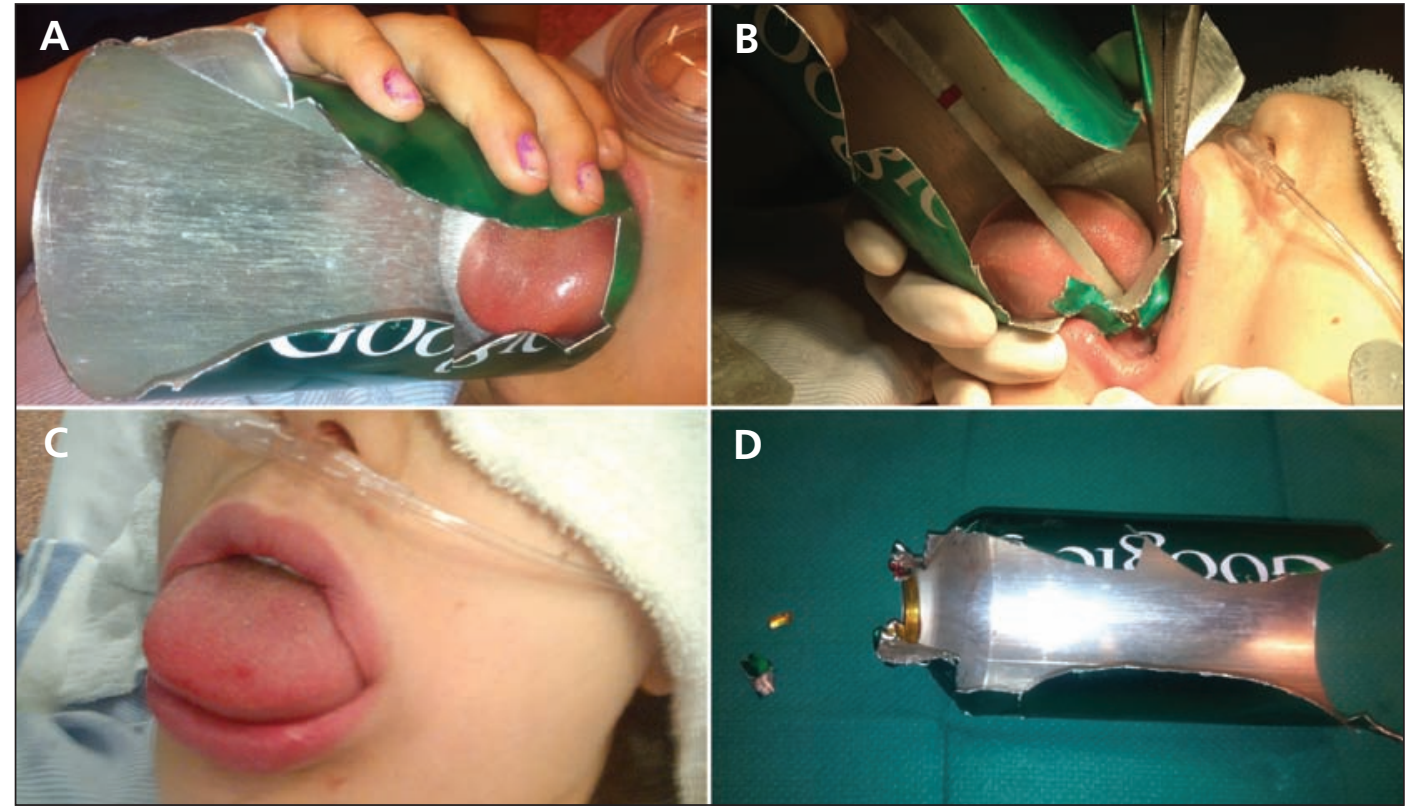

Figure 1: The edematous tongue of a 10-year-old girl during removal of the metal bottle in which it was entrapped ( $A$ and $B$ ) and immediately after surgery (C). The restricting brass ring and metal bottle (D) were removed with tinsnips and a ring cutter.

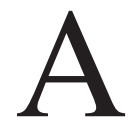
10-year-old healthy girl got her tongue stuck in a metal water bottle when she placed her tongue inside and sucked out air, creating a vacuum effect. Following unsuccessful attempts at removal by emergency medical services and an otolaryngologist (who broke the vacuum seal by removing the base), she was transferred to a pediatric centre. Her airway remained stable, and spontaneous ventilation was supported with oxygen via nasal prongs. With preparations made for possible fiber-optic nasal intubation and tracheotomy, ketamine and glycopyrrolate were given intravenously. The patient remained cooperative and calm, and her airway was stable throughout the procedure.

The mouth of the bottle consisted of a thick brass ring under the aluminum, and its removal required the use of tinsnips, a ring cutter and malleable retractors (Figure 1, B). After surgery, the patient was monitored in the intensive care unit because of the tongue edema, but she did not require intubation. Her tongue remained swollen and initially could not be retracted fully into her mouth. Dexamethasone was given intravenously, and the edema decreased substantially the next day. On postoperative day two, the patient had regained full movement of her tongue, with minimal edema, normal swallowing and little pain. Several weeks later, she remained asymptomatic and had normal tongue mobility.

Unsuccessful removal attempts following tongue entrapment in aluminum water bottles can aggravate pain and swelling due to venous congestion and ischemia and may lead to airway compromise. Similar cases have been reported in the literature, ${ }^{1,2}$ and parents should be made aware of this unusual but dangerous situation.

\section{References}

1. Shah G, Sciarrino J, Barth P, et al. Tongue entrapment in aluminum water bottle: discussion of removal and airway management. Int J Pediatr Otorhinolaryngol 2012;76:757-60.

2. Whited CW, Rocke DJ, Lee WT. Tongue entrapment in metal drinking bottle. Arch Otolaryngol Head Neck Surg 2011;137:625-7.
Competing interests: None declared.

The authors have obtained patient consent.

This article has been peer reviewed.

Affiliations: Department of Otolaryngology - Head \& Neck Surgery (Fernandes, Campisi), Department of Anesthesia (Ng), University of Toronto; Department of Anesthesia (Ng),

Department of

Otolaryngology - Head \& Neck Surgery (Campisi),

Hospital for Sick Children, Toronto, Ont.

\section{Correspondence to:}

Vinay Fernandes,

vinay.fernandes@mail

.utoronto.ca

CMAJ 2014. DOI:10.1503

/cmaj.140112 